Www.jmscr.igmpublication.org

Impact Factor (SJIF): 6.379

Index Copernicus Value: 79.54

ISSN (e)-2347-176x ISSN (p) 2455-0450

crossrefDOI: https://dx.doi.org/10.18535/jmscr/v7i1.58

Journal Of Medical Science And Clinical Research

\title{
Pure Accelerated Radiotherapy with Concurrent Chemotherapy in State III and IV Head \& Neck Squamous Cell Carcinoma
}

Authors

\section{Vijayasree Thirukurungudi Narayanan ${ }^{1}$ Saravanan Subramaniyan ${ }^{2}$}

${ }^{1}$ Associate Professor, Government Royapettah Hospital \& Kilpauk, Medical College, Chennai

${ }^{2}$ Professor, Government Royapettah Hospital \& Kilpauk, Medical College, Chennai

Corresponding Author

Vijayasree T N

Associate Professor Govt Royapettah Hospital \& Kilpauk Medical College, Chennai, Tamilnadu, India Email: tnvijayasree@gmail.com,9444132816

\begin{abstract}
Background: Stage III-IVB Squamous cell carcinoma in the head \& neck region (HNSCC) has been one of the foremost problems among oncologists to achieve good results, even with Combined modality approach. Chemotherapy with radiotherapy in different combinations and different schedules, has been tried by many researchers to achieve good loco-regional control, among those not amenable for surgery. Objective of this study, is to determine the loco regional response and acute toxicity patterns when combining Pure Accelerated Radiotherapy (6 Fractions $(F x)$ /week) with Concurrent Chemotherapy in patients with advanced loco regional HNSCC.

Methods: Patients presented to our Department with previously untreated stage III - stage IV HNSCC with age between 18yrs and 60 yrs of either gender, without systemic spread, were included in our study. They were treated with radical external radiation $6600 c G y / 33(F x), 200 c G y / F x, 6 F x /$ Week over 5.3 weeks (38 days) and concurrent chemotherapy Cisplatin $\left(100 \mathrm{mg} / \mathrm{m}^{2}\right)$ administered intravenously on days 1 and 22 of Radiotherapy. The primary end-point of the study was the loco regional response at 6 weeks. Additional end point includes acute toxicity rate.

Results: Among 28 patients recruited, 25 patients completed full course of treatment. 21 patients (84\%) had complete response in and $4(16 \%)$ had partial response, among them, 3(12\%) patients had residual disease at nodal site, and 1(4\%) at the primary and nodal site. $7(28 \%)$ and $17(70.8 \%)$ patients experienced acute Grade-III and grade-II side effects respectively.

Conclusions: This data shows that it is feasible to combine Accelerated Radiotherapy and full dose monochemotherapy with manageable, although substantial, toxicity. The hospital stay is reduced. Hence it is an effective alternate regimen in centers with high work load.

Keywords: Squamous cell carcinoma, Head and neck cancers, concurrent chemoradiation, Altered Fractionation.
\end{abstract}

Introduction

Squamous cell carcinoma in the head and neck region (HNSCC) has been one of the foremost challenges among oncologists to achieve good results, concerning, the treatment and complications. The habit of tobacco and betel nut chewing, cigarette smoking along with alcohol ingestion remains as the etiology of these cancers, 
which is the common habit of many people. To compound the problem $70-80 \%$ of cases coming to our Institute for treatment are in Stage III-IVB, where management is difficult, and the prognosis is poor.

At our Department of Radiation Oncology, we register around 2500-2800 new cancer patients annually. Of this patient load, 30\% are head and neck cancers (HNSCC), nearly $15-20 \%$ being cancers of the Oropharynx, Larynx, and Hypopharynx. 65-80\% of the HNSCC present in the locally advanced stage (Stage III-IVB,) at our institution.

Treatment for Locally Advanced HNSCC is Stage III - IVB is Combined modality. Options available are

(1) Surgery + Postoperative Radiation therapy.

(2) Altered Fractionation.

(3) Chemo-Radiation.

Progress in surgical resection - reconstructive techniques and advances in radiotherapy treatment planning and delivery methods yield a good outcome in the majority of patients with early HNSCC. Unfortunately, for more locally advanced cancers, the current standard therapy consisting of surgical resection and Pre or Postoperative Radiotherapy, still achieves rather poor results regarding disease control, organfunction preservation, or both. Consequently, there has been a continuous search for better treatment approaches.

Advances in radiobiologic concepts have led to the development of new fractionation schedules in radiation therapy, Altered Fractionation for the treatment of head and neck cancers. They are Hyper fractionation and Accelerated fractionation. In accelerated radiation therapy, the existing trials can be grouped into two categories; Pure Accelerated fractionation and Hybrid Accelerated fractionation. Multiple studies have tested hyper fractionation and various accelerated fractionation regimens with positive results ${ }^{[1]}$. To further improve the locoregional control, various modalities like chemoradiation, radiosensitizers, and hyperthermia can be used. Concurrent
Chemo-irradiation strategies have emerged in the horizon of head and neck cancer treatment, and are now replacing traditional modalities in the locally advanced clinical situation as a standard of care. This gives adequate locoregional control with organ with function preservation. Chemotherapy administered concurrently with radiation has improved the 2 and 5-year overall survival rates by $8 \%,{ }^{[2]}$ but, unfortunately, at the expense of increased toxicity. ${ }^{[3]}$ The addition of some single agents to radiation has improved response rates at the cost of additional toxicity. ${ }^{[4,5,6]}$ The data of five recent trials addressing standard radiation fractionation with or without cisplatin have been reported. ${ }^{[7-11]}$ All five trials showed superior outcome in favor of the combined regimen regardinglocoregional control or organ preservation, and three trials also showed improvement in survival. ${ }^{[2],[7],[9],[10]}$

A nonrandomizedsingle-institution study was performed at Charing Cross \& Hammersmith Hospitals NHS Trust, London ${ }^{[12]}$ to study the feasibility of combining accelerated radiotherapy with chemotherapy. In this study, radical radiotherapy was performed according to guidelines of DAHANCA $7^{[13]}$, along with, Cisplatin chemotherapy $75 \mathrm{mg} / \mathrm{m}^{2}$ with hydration was scheduled on day 1 and day 22 of radiotherapy. The results, the addition of platinum chemotherapy to accelerated radiotherapy is feasible, disease-free survival at 2years was $67 \%$; acute toxicity was significant and manageable is the basis of the present study.

\section{Aim}

If this study is to determine thelocoregional response and acute toxicity patterns when combining Pure Accelerated Radiotherapy (6 Fractions $(\mathrm{Fx}) /$ week $)$ with Concurrent Chemotherapy using Cisplatin $100 \mathrm{mg} / \mathrm{m}^{2}$ in patients with advanced locoregional HNSCC. The primary end-point of the study was the locoregional response at 6 weeks. Additional endpoint includes acute toxicity rate. 


\section{Materials and Methods}

This Prospective study was conducted in patients presented to our Department with locally advanced and unresectable (either UICC stage III or stage IV A), previously untreated squamous cell carcinoma of the oropharynx, hypopharynx, and larynx with age between 18yrs and 60 yrs of either gender, Performance status ECOG 0-1 and life expectancy of more than a year, without systemic spread, with adequate renal function and hemoglobin were included in our study. Informed consent form signed before study entry was got.

All patients are persuaded to quit smoking and alcohol. Dental evaluation and management if needed done. Feeding tubes (NG tube/ Feeding Gastrostomy/ Feeding Jejunostomy tube) placed for nutrition if needed.

They are treated with intent radical external radiation $6600 \mathrm{cGy} / 33 \mathrm{Fx}, 200 \mathrm{cGy} / \mathrm{Fx}, 6 \mathrm{Fx} /$ Week over 5.3 weeks (38 days) and concurrent chemotherapy Cisplatin $\left(100 \mathrm{mg} / \mathrm{m}^{2}\right)$ administered intravenously on days 1 and 22 of Radiotherapy.

Radiation therapy (RT) is given as pure accelerated RT using Megavoltage equipment, Cobalt-60 unit, Thearatronics,780 Phoenix, Canada was used to provide appropriate photon energies. Treatment commences on Monday continued up to Saturday, Sunday is the rest day, to a total of $6600 \mathrm{cGy} / 33 \mathrm{Fx}, 200 \mathrm{cGy} / \mathrm{Fx}$ over 5.3 weeks (38 days). An unexpected holiday in a week is compensated by giving an extra fraction on any one weekday 6 hours after the first fraction. Primary and involved nodes should receive a total dose of $6600 \mathrm{cGy}$, uninvolved draining lymph nodes should receive a dose of 5000 cGy. The primary field must be reduced at 40 Gy to off the spinal cord. Target Volume includes primary tumor and known or suspected lymph node disease and treated with lateralopposed field with a margin. Initial field is shifted at 40 Gy to limit dose to the spinal cord after 50 Gy field is reduced again to include only the primary and involved lymph nodes up to 6600 cGy. Our Department uses2D planning. Patient positioned, Supine with neck rest and shoulder retraction and reproducibly immobilized. Treatment Verification is done with the patient in the treatment position, simulation films (Fig.1) are taken, Treatment portals verified and corrections made if necessary. Maximum dose to the spinal cord is kept as $45 \mathrm{~Gy} / 25 \mathrm{fx} / 4.1$ weeks.

\section{Chemotherapy - Cisplatin Dose Schedule}

Patients received Cisplatin $\left(100 \mathrm{mg} / \mathrm{m}^{2}\right)$ administered intravenously on days 1 and 22 .

Ondansetron $16 \mathrm{mg}$ intravenously (i.v) will be given 30 minutes before cisplatin chemotherapy as premedication. Any pre-existing dehydration corrected before cisplatin administration. Patients received vigorous hydration and diuresis. The regimen followed was; pre-hydration with a $1000 \mathrm{ml}$ of D5NS over 2-4 hours. Inj. Mannitol $12.5 \mathrm{~g}$ i.v.was given bolus before cisplatin. Cisplatin $100 \mathrm{mg} / \mathrm{m}^{2}$ in $500 \mathrm{ml} \mathrm{NS}$ over $1-2$ hours with post-hydration as clinically indicated.

\section{Dose Modifications for day 22 Cisplatin:}

If on the day of scheduled treatment with cisplatin the absolute neutrophil count $(A N C)$ is $<1000$, the platelet count is $<75,000$ treatment; itis held until ANC and platelet count becomes more than 1000 and 75000 respectively. Creatinine Clearance should be more than $50 \mathrm{ml} / \mathrm{min}$ to receive a dose of $100 \mathrm{mg} / \mathrm{m}^{2}$ of cisplatin. If any signs of paralysis, moderate myopathy, moderate weakness, seizure or peripheral neuropathy occur, cisplatin was to be discontinued.

\section{Supportive Care}

Placement of a gastrostomy tube (PEG or PFG) before treatment begins was strongly counseled to optimize nutrition and hydration during combined therapy, or else Naso-gastric feeding was opted for. Aggressive oral and skin care is given and analgesics and other symptomatic support given.

\section{Toxicity Reporting}

The revised NCI Common Toxicity Criteria (CTC) Version 3.0 was used to score all chemotherapy and acute radiation ( $\leq 90$ days) 
toxicities associated with this protocol, except for skin and mucosa, for which RTOG criteria was used (Grade I to IV). Radiation toxicities appearing or persisting beyond 90 days from the start of protocol treatment were evaluated using the RTOG Late Toxicity Scoring Scheme.

\section{Acute Reactions}

The local reaction of skin and mucous membranes was scored at least weekly during radiotherapy and post-therapy until clearance. Response of tumor documented weekly during therapy and at each follow-up. Documentation of the size of the tumor is by caliper or ruler, measuring the longest measurement and at right angles to it, by inspection and palpation.

\section{Response Criteria for Measurable Lesions}

Complete Response (CR)- Complete disappearance of measurable and palpable disease.

Partial Response(PR) - Tumor shrinkage greater than $50 \%$ of the product of the perpendicular diameters of the two largest dimensions without an increase in the size of any other area of known malignant disease (excluding regional nodes) or without the appearance of new areas of malignant disease within the treated volume.

No change(NC) - Up to $25 \%$ growth or $25 \%$ shrinkage of the product of perpendicular diameters of the two largest dimensions without an increase in the size of any other area of known malignant disease (excluding regional nodes) or without the appearance of new areas of malignant disease within the treated volume.

Progression (P) - Growth of tumor greater than $25 \%$ of the product of the perpendicular diameters of the two largest dimensions

\section{Response Criteria for Evaluable, Non- Measurable Lesions \\ Complete Response (CR)- Complete disappearance of known disease}

Partial Response (PR)- Definite decrease in tumor size. This should be confirmed by at least two investigators evaluating independently, or photographs or x-rays should be submitted for review.

Patients were inpatients and underwent weekly examination during treatment. On completion of the treatment, evaluation is done at first follow-up (6 weeks). Subsequently, patients are being assessed every 3 months for the first 2 years and every 6 months for years 3 to 5 , and annually thereon. In addition to the tumor and clinical status, acute toxicity was graded. Systemic and acute radiation effects were scored using the National Cancer Institute Common Toxicity Criteria version 3.0.

The primary end-point of the study was the locoregional response at 6 weeks. Additional endpoint includes acute toxicity rate. Apart from describing the distribution of different variables; chi-square test, cross-tabulation and percentage analysis were applied to the data available to determine the significance and relationship between the variables.

\section{Results}

28 patients met the eligibility criteria of the protocol and were recruited. Three patients were excluded from the study because they opted out of the protocol therapy early on. Table 1 lists pretreatment patient and tumor characteristics.

Some significant observations noted were that dysphagia and odynophagia were common troublesome symptoms in patients with oropharynx, while voice change was the troublesome symptom among patients with laryngeal cancer; in hypopharyngeal cancers, the troublesome symptoms were swelling, pain and dysphagia, $(\mathrm{p}<0.01)$. Nasogastric tube feeding was required before treatment increasingly with cancers of the hypopharynx and oropharynx.

The fractionation regimen was according to protocol specification in all patients $(100 \%)$. The duration of Radiation therapy was $\leq 39$ days in 23 patients $(92 \%)$ and was 40 days in 1 patient $(4 \%)$. All the patients received both cycles of Cisplatin. Dose modification for the second cycle was required in 2 patients because of toxicity. All 
patients received therapy as per protocol or acceptable variations for both radiation and chemotherapy.

Overall response to therapy was recorded in 25 patients (100\%). This included a complete response in 21 patients $(84 \%)$ and partial response in 4 patients (16\%). No patient (0\%) had progressive disease. Of the 4 patients with residual disease (partial response), 3(12\%) patients had residual disease at nodal site, and $1(4 \%)$ patient had residual at the primary and nodal site. Table 2 shows the response distribution and stage wise response.

\section{Acute Toxicity}

7 patients (28\%) experienced Grade-III side effects, and 17 patients $(70.8 \%)$ had acute grade-II side effects. The most common acute grade 3 side effects in decreasing frequency were mucositis and hematological toxicity. Grade 3 side-effects commonly occurred in relation to mucositis, hematological toxicity, nausea/ vomiting, and dysphagia. Table 3 lists the type and frequency of side-effects.

There is a significant association between the toxicities observed and suspension of treatment (esp. irradiation) $(\mathrm{p}<0.05)$. There was no significant relationship between the toxicities observed and the site and stage of disease, and type of response.

Table 1 Distribution of Patient and Tumor Characteristics

\begin{tabular}{|c|c|c|}
\hline Variable & No. of Patients & Percentage \\
\hline AGE (years) & & \\
\hline Median & 53 years & \\
\hline Range & $40-60$ years & \\
\hline SEX & & \\
\hline Male & 24 & 96 \\
\hline Female & 01 & 4 \\
\hline HABITS & & \\
\hline Smoking & 23 & 92 \\
\hline Alcohol abuse & 17 & 68 \\
\hline Tobacco chewing & 4 & 16 \\
\hline Troublesome Longstanding & & \\
\hline Symptoms & 20 & 80 \\
\hline Dysphagia & 10 & 40 \\
\hline Voice Change & 02 & 08 \\
\hline Odynophagia & 13 & 52 \\
\hline Pain & 08 & 32 \\
\hline Swelling & & \\
\hline SITE & & \\
\hline Oropharynx & 15 & 60 \\
\hline Hypopharnx & 07 & 28 \\
\hline Larynx & 03 & 12 \\
\hline AJCC Stage Grouping & & \\
\hline Stage III & 10 & 40 \\
\hline Stage IVA & 15 & 60 \\
\hline GRADE (Squamous Cell Carcinoma) & & \\
\hline Grade- I & 05 & 20 \\
\hline Grade- II & 14 & 56 \\
\hline Grade- III & 02 & 08 \\
\hline
\end{tabular}


Table 2: Distribution of Tumour Response

\begin{tabular}{|c|c|c|}
\hline Particulars & $\begin{array}{c}\text { Complete } \\
\text { Response N } \\
(\%)\end{array}$ & $\begin{array}{c}\text { Partial Response } \\
\mathbf{N}\end{array}$ \\
\hline Overall Response & $21 / 25 \quad(84)$ & $4 / 25$ \\
\hline $\begin{array}{l}\text { Grade-wise } \\
\text { Grade-I } \\
\text { Grade-II } \\
\text { Grade-III } \\
\text { P-Value }\end{array}$ & \begin{tabular}{ll}
$5 / 5$ & $(\mathbf{1 0 0})$ \\
$10 / 14$ & $\mathbf{( 7 8 . 6 )}$ \\
$2 / 2$ & $\mathbf{( 1 0 0 )}$ \\
\multicolumn{2}{c}{$\mathbf{0 . 6 4 5}$}
\end{tabular} & \begin{tabular}{cc}
0 & $(0)$ \\
$4 / 14$ & $(\mathbf{2 1 . 4 )}$ \\
0 & $(\mathbf{0})$ \\
\multicolumn{2}{c}{$\mathbf{0 . 5 9 7}$}
\end{tabular} \\
\hline $\begin{array}{l}\text { Site-wise } \\
\text { Oropharynx } \\
\text { Larynx } \\
\text { Hypopharynx } \\
\text { P-Value }\end{array}$ & \begin{tabular}{lr}
$11 / 15$ & $(\mathbf{7 3 . 4 )}$ \\
$3 / 3$ & $(\mathbf{1 0 0 )}$ \\
$7 / 7$ & \multicolumn{1}{c}{$(\mathbf{1 0 0 )})$} \\
\multicolumn{2}{c}{$\mathbf{0 . 3 2 1}$}
\end{tabular} & 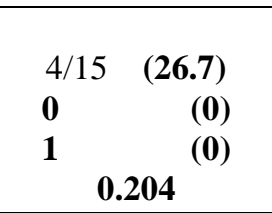 \\
\hline $\begin{array}{l}\text { Stage-wise } \\
\text { Stage-III } \\
\text { Stage-IVA } \\
\text { P-Value }\end{array}$ & 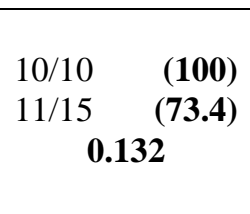 & $\begin{array}{cc}\mathbf{0} & (\mathbf{0}) \\
4 / 15 & (\mathbf{2 6 . 4}) \\
\mathbf{0 . 0 7 5} & \end{array}$ \\
\hline
\end{tabular}

Table 3: Type and Frequency of Acute Side effects Observed in 24 patients

\begin{tabular}{|l|c|c|c|c|c|}
\hline & \multicolumn{5}{|c|}{ No. of Patients (n=24) } \\
\hline Toxicity & Grade I & Grade II & Grade III & Grade IV & Grade V \\
\hline Hematologic & $11(45.8 \%)$ & $7(29.2 \%)$ & $3(12.5 \%)$ & - & - \\
\hline Infection,Febrile Neutropenia & - & - & $2(8.3 \%)$ & - & - \\
\hline Renal Function & $1(4.2 \%)$ & $2(8.3 \%)$ & - & - & - \\
\hline Dysphagia & - & $18(75.0 \%)$ & $3(12.5 \%)$ & - & - \\
\hline Mucositis & $9(37.5 \%)$ & $14(58.3 \%)$ & $2(8.3 \%)$ & - & - \\
\hline Nausea/Vomiting & $18(75.0 \%)$ & $4(16.7 \%)$ & $1(4.2 \%)$ & - & - \\
\hline Auditory & $2(8.3 \%)$ & $1(4.2 \%)$ & - & - & - \\
\hline Skin & $8(33.3 \%)$ & $15(62.5 \%)$ & - & - & - \\
\hline Pain & $8(33.3 \%)$ & $14(58.3 \%)$ & $3(12.5 \%)$ & - & - \\
\hline
\end{tabular}

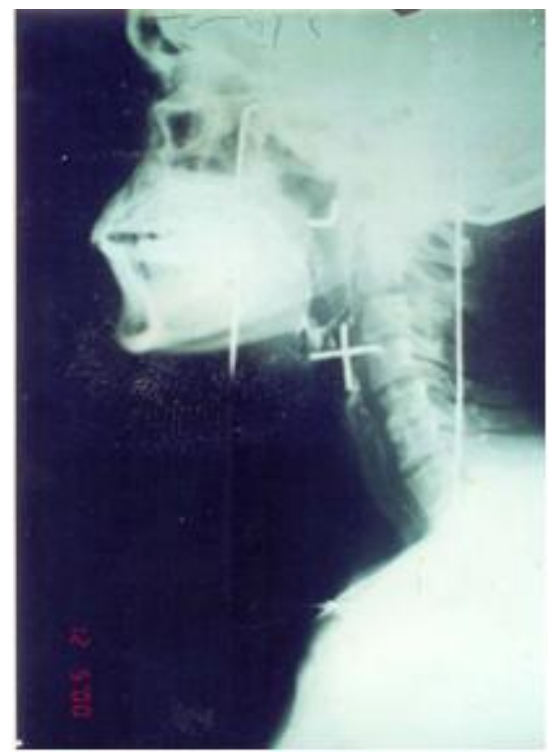

Fig.1 Simulation --- Oropharynx

\section{Discussion}

The findings that some modified radiation fractionation and concurrent chemoradiation regimens are more effective than conventionally fractionated radiation therapy in the treatment of advanced HNSCC, generated interest to test the combination of altered fractionation regimens with chemotherapy. The results of six phase III trials testing the efficacy of such combinations of altered fractionation regimens with concurrent chemotherapy against radiation alone have been reported. The radiation regimens used were accelerated fractionation in three trials, ${ }^{[14],[15]}$ hyperfractionation in one study, ${ }^{[16]}$ and splitcourse altered fractionation in two trials. ${ }^{[17],[18],[19]}$ Collectively, most trials show that combinations of modified fractionation regimens with chemotherapy achieve better local control and, in several trials, improved survival compared with standard or altered fractionation alone. However, the value of altered fractionation in the concurrent 
chemoradiation setting (i.e., the potential benefit of combining altered fractionations instead of standard fractionation with chemotherapy) has not been tested. Building on the results of RTOG 9003, which show locoregional tumor control benefit by concomitant boost regimen $(\mathrm{ACB})^{[20]}$, RTOG 99-14 undertook a phase-II trial to determine the feasibility of delivering two cycles of Cisplatin $\left(100 \mathrm{mg} / \mathrm{m}^{2}\right)$ on days 1 and 22 of ACB in a cooperative group setting. This study shows an estimated $2 \mathrm{yr}$ overall survival and disease-free survival of $71.6 \%$ and $53.3 \%$, respectively. The complete response rates in this study were $83 \%$, and acute grade- 4 and grade- 3 toxicity rates were $25 \%$ and $64 \%$ respectively ${ }^{[21]}$. There are two randomized phase III trials which addressed the issue of 6 fractions per week. In DAHANCA $6 \& 7$ there was a significant improvement in the locoregional control. Another study by D. Power et al., showed disease-free survival at $2 \mathrm{yr}$ as $67 \%$, on combining Inj.cisplatin $75 \mathrm{mg} / \mathrm{m}^{2}$ on days $1 \&$ 22 with the above regimen. This study was based on the DAHANCA 7 and study by D. Power et al., but in the setting of a developing country and a Telecobalt treatment facility. All the patients completed the treatment, both radiation therapy and chemotherapy as specified or with minor variations.

In this study, patients were treated with 6 fractions per week to a total dose of 66Gy which reduced the overall treatment time by 1 week. Withers et al., and Bentzen and Thames showed that a dose of 0.48 Gy per day was recovered by a tumour during fractionated radiotherapy of HNSCC. This was the reason why in our study in which overall treatment time was reduced by 1 week, produced a higher response than conventional fractionation. By reducing overall treatment time by 1 week, the 'Dose recovery factor' of 3.3Gy was avoided. The concept of effective doses was suggested by Jack Flower based on the LQ model. Based on the calculations we have delivered $72.4 \mathrm{~Gy}$ in 5.3 weeks, which is equal to $70 \mathrm{~Gy}$ in conventional fractionation in 7 weeks.
In the Danish trial, the impact of the reduction in overall treatment time was shown in well and moderately differentiated tumours. There was a $27 \%$ improvement in the tumour control when the overall treatment time was reduced to 5.3 weeks. In our study, the complete enhanced response in well and moderately differentiated tumourswas concurring with DAHANCA 7 trial. The better response shown with poorly differentiated tumours in our study may be due to the addition of chemotherapy.

When the subset analysis was performed, it showed a better response rate in all subsites, although it was significant in hypopharyngeal tumours in our study.

When we analyzed by $\mathrm{T}$ size and $\mathrm{N}$-stage, our study produced good response rates. It was significant for T3 tumours and N0, N1, N2a, N2b stages. The Danish study reported that the whole benefit of acceleration came from improved $\mathrm{T}$-site control, which further improved with chemotherapy. We also noted that when T-size or $\mathrm{N}$-stage increases, the response rate comes down, which was shown by Stanley et al. They postulated that it could be due to poor internal vascularity, necrosis, and hypoxia.

Finally, all stages showed improved response with acceleration. The addition of chemotherapy has an added advantage.

Since the early responding tissues have $\alpha / \beta$ ratio as that of squamous cell carcinoma of the head\& neck, it responds similarly to a tumour. In our study, most of the reactions are Gr-I \& II (Skin Gr-II 66\%, Gr-I $32 \%$ : Mucosal Gr-II 56\%, Gr-I $36 \%)$. Only2 patients developed Gr-III mucositis, which started at the end of $4^{\text {th }} \mathrm{wk}$ and peaked at $5^{\text {th }}$ week. All these did not result in treatment break.

In the Danishtrial, the reported percentage of confluent mucositis was 53\%. Jackson et al., reported $27 \%$ of severe mucositis with accelerated radiotherapy, whereas $62 \%$ of high-grade mucositis was reported by Skladowski. Comparing the acute toxicity with trials conducted by several institutions, however, is rather difficult because of inconsistency in recording and 
reporting, as clearly pointed out by Trotti and Bentzen $^{[22]}$. These authors noted that four different recognized grading systems and two descriptive efforts had been used in reporting the results of nine frequently cited trials addressing the combination of radiation and chemotherapy HNSCC published within the last decade. Comparison of the results of this study with these other trials revealed similar incidences of grade 3 and grade 4 adverse effects.

\section{Conclusion}

In conclusion, this data shows that it is feasible to combine Accelerated Radiotherapy and full dose mono-chemotherapy using cisplatin with manageable, although substantial, toxicity. The compliance to therapy is high, and the locoregional response achieved compared favorably with Accelerated Radiotherapy alone or other concurrent chemoradiation regimens available in the literature. The hospital stay is reduced. Hence it is an effective alternate regimen in centers with the highworkload. An extended phase II trial and a new Phase-III trial comparing Accelerated radiotherapy plus cisplatin against standard radiation plus cisplatin is being planned at the institute to determine whether the use of Accelerated Radiotherapy in the concurrent chemoradiation setting further improves outcome.

Sources of support in the form of grants: Nil

\section{References}

1. Ang K.: Altered Fractionation in Head and Neck Cancer. Seminars in Radiation Oncology. 8:230-236, 1998.

2. Pignon JP, Bourhis J, Domenge C, et al: Chemotherapy added to locoregional treatment for head and neck squamous-cell carcinoma: Three meta-analyses of updated individual data. Lancet 355:949955, 2000.

3. El-Sayed S., Nelson N.: Adjuvant and adjuctive chemotherapy in the management of squamous cell carcinoma of the head and neck region. A metaanalysis of prospective and randomised trials. Journal of Clinical Oncology. 14:838-847, 1996.

4. Marcial V.A., Pajak T.F., Mohiuddin M., Cooper J.S., Al-Sarraf M., Mowry P.A., Curran W., Crissman J., Rodriguez M., Velez-Garcia E.: Concomitant cisplatin chemotherapy and radiotherapy in advanced mucosal squamous cell carcinomas of the head and neck. Longterm results of the Radiation Therapy Oncology Group study 81-17. Cancer. 66:1861-1868, 1990.

5. Koch W.M., Lee D.J., Eisele D.W., Miller D., Poole M., Cummings C.W., Forastiere A.: Chemoradiotherapy for organ preservation in oral and pharyngeal carcinoma. Archives of Otolaryngology Head and Neck Surgery.121:974 -980, 1995.

6. Byfield J., Sharp T., Frankel S., Tang S., Callipari F.: Phase I and II trial of five-day infused 5-fluorouracil and radiation in advanced cancer of the head and neck. Journal of Clinical Oncology.2:406 - 413, 1984.

7. Al-Sarraf M, LeBlance M, Shanker PG, et al: Chemoradiotherapy versus radiotherapy in patients with advanced nasopharyngeal cancer: Phase III randomized intergroup study 0099. J ClinOncol 16:1310-1317, 1998

8. Adelstein DJ, Li Y, Adams GL, et al: An intergroup phase III comparison of standard radiation therapy and two schedules of concurrent chemoradiotherapy in patients with unresectable squamous cell head and neck cancer. J Clin Oncol 21:92-98, 2003.

9. Forastiere AA, Goepfert H, Maor M, et al: Concurrent chemotherapy and radiotherapy for organ preservation in advanced laryngeal cancer. N Engl J Med 349:2091-2098, 2003 
10. Cooper JS, Pajak TF, Forastiere AA, et al: Post-operative concurrent radiotherapy and chemotherapy for high-risk squmaous cell carcinoma of head and neck. N Engl J Med 350:1937-1944, 2004.

11. Bernier DM, Domenge C, Ozsahin M, et al: Postoperative irradiation with or without concomitant chemotherapy for locally advanced head and neck cancer. $\mathrm{N}$ Engl J Med 350:1945-1952, 2004.

12. Power D, Alonzi R, Marcou Y, Eales C, et al: Combined chemotherapy with accelerated radiotherapy in the radical management of locally advanced headsneck malignancy. A feasibility study Intl J Radiation Oncol Biol Physics Vol 60, Number 1, Supplement 2004

13. Overgard J, Sand Hansen H, et al: Five compared with six fractions per week of conventional radiotherapy of HNSCC. Report of DAHANCA Trial 6\&7 : The Lancet 2003: 362; 933-40

14. Staar S, Rudat V, Stuetzer H, et al: Intensified hyperfractionated accelerated radiotherapy limits the additional benefits of simultaneous chemotherapy. Results of a multicentre randomized German trial in advanced head and neck cancer.Int $\mathbf{J}$ Radiat Oncol Phy 50:1161-1171, 2001.

15. Bourhis J, Lapeyre M, Tortochaux J, et al: Preliminary results of the GORTEC 96-01 randomized trial comparing very accelerated radiotherapy versus concomitant radio-chemotherapy for locally inoperable HNSCC. Int J Radiat Oncol Biol Phys 51:39, 2001 (suppl)

16. Jeremic B, Shibamoto Y, Milicic B, et al: Hyperfractionated radiation therapy with or without concurrent low-dose daily cisplatin in locally advanced squamous cell carcinoma of the head and neck: A prospective randomized trial. J ClinOncol 18:1458-1464, 2000.

17. Wendt TG, Grabenbauer GG, Rodel CM, et al: Simultaneous radiochemotherapy versus radiotherapy alone in advanced head and neck cancer: A randomized multicentre study. J ClinOncol 16:13181324

18. Calais G, Alfonsi M, Bardet E, et al: Randomized trial of radiation therapy versus concomitant chemotherapy and radiation therapy for advanced stage oropharynx carcinoma. J Natl Cancer Inst 91: 2081-2086, 1999.

19. Bridget GP, Kwok B, Baldwin M, et al: Craniofacial resection for paranasal sinus cancers. Head Neck 22: 772-780, 2000

20. Fu KK, Pajak TF, Trotti A, et al: A Radiation Therapy Oncology Group (RTOG) Phase III randomized study to compare hyperfractionated and two variants of accelerated fractionation to standard fractionation radiotherapy for Head and Neck squamous cell carcinomas: First report of RTOG 9003.Intl J Radiation OncolBiol Physics 48:7-16, 2000.

21. Ang KK, Harris J, Garden AS, et al: Concomitant boost radiation plus concurrent cisplatin for advanced head and neck carcinomas: radiation therapy oncology group phase II trial 99-14. J ClinOncol 23: 3008-3015, 2005.

22. Trotti A, Bentzen SM: The need for adverse effects reporting standards in oncology clinical trials. J ClinOncol 22: 19-22, 2004. 